\title{
Diagnostic accuracy of urinary latex agglutination test (KAtex) for the diagnosis of visceral leishmaniasis: A meta-analysis
}

\author{
Mohammad Fararouei ${ }^{1}$, Bahador Sarkari ${ }^{2,3}$, Samaneh Abdolahi Khabisi ${ }^{4}$, Zahra Rezaei $^{2}$ \\ ${ }^{1}$ Department of Epidemiology, School of Public Health, Shiraz University of Medical Sciences, Shiraz, Iran \\ 2 Department of Parasitology and Mycology, School of Medicine, Shiraz University of Medical Sciences, Shiraz, Iran \\ ${ }^{3}$ Basic Sciences in Infectious Diseases Research Center, Shiraz University of Medical Sciences, Shiraz, Iran \\ ${ }^{4}$ Department of Parasitology and Mycology, Faculty of Medicine, Zahedan University of Medical Sciences, \\ Zahedan, Iran
}

\begin{abstract}
Latex agglutination test (KAtex) has been used in the last two decades for the diagnosis of visceral leishmaniasis (VL) in different VL-endemic areas. Here, we present a meta-analysis of studies which evaluated the KAtex for the diagnosis of VL to find out its overall diagnostic performance. A database search was performed on PubMed, Scopus, ISI Web of Science, Iranmedex and Google Scholar. The search of databases found 57 papers, of which 17 articles fulfilled our eligibility criteria. Meta-analysis of diagnostic accuracy (MADA) and Hierarchical Summary Receiver Operating Curve (HSROC) packages were used to do the meta-analysis and to obtain pooled estimates of sensitivity and specificity. Fixed effect bivariate analysis was conducted, using Mantel-Haenszel estimator, to measure the performance and diagnosis odds ratio (DOR) of the test. Heterogeneity of the test results was assessed by Chi-squared test.

The sensitivity of individual studies ranged from 39.8 to $100 \%$, and the specificity ranged from 64 to $100 \%$. The combined sensitivity and specificity estimates of KAtex were 77\% (95\% CI, 70-83\%), and 97\% (95\% CI, 93-97\%), respectively. Comparing the performance of the test by region suggests a significant difference where the lowest and highest sensitivities are reported from Nepal/Tunisia and Europe/Middle East respectively $(p<0.05)$. On the other hand, the lowest and highest rates of specificity were reported from Sudan and America/Middle East respectively.

The overall specificity of KAtex is satisfactory. However, KAtex suffers from low sensitivity and this shortcoming should be improved. The test provides a rapid and simple diagnosis of VL and improvement of its sensitivity deserve further studies.
\end{abstract}

Keywords: KAtex; diagnosis; visceral leishmaniasis; meta-analysis.

J Infect Dev Ctries 2018; 12(12):1045-1051. doi:10.3855/jidc. 10185

(Received 16 January 2018 - Accepted 20 October 2018)

Copyright (C) 2018 Fararouei et al. This is an open-access article distributed under the Creative Commons Attribution License, which permits unrestricted use, distribution, and reproduction in any medium, provided the original work is properly cited.

\section{Introduction}

Visceral leishmaniasis (VL) as a protozoan parasitic infection is a significant health problem in several countries of the world [1-4]. The disease is fatal in more than $95 \%$ of cases, if left untreated. Accurate diagnosis of VL is crucial for proper management of the disease. Several immunodiagnostic tests, with different sensitivity and specificity, have been developed for the diagnosis of VL [5-9]. Among them, tests which are based on detection of antigen in sera or urine received special attention. The most studied and the only antigen-based latex agglutination test for the diagnosis of VL is the Latex Agglutination Test (KAtex) which detects a $5-15 \mathrm{kDa}$ low molecular weight carbohydrate antigen in the urine of VL patients $[10,11]$. The test was originally developed by Attar at Liverpool School of Tropical Medicine, UK, in 2001 [10] and soon after that was commercialized by Kalon Biological Ltd (Guildford, UK), known as KAtex. KAtex is a test of cure which detects only patients with active disease, and rapidly turns negative after successful treatment. The target antigen for KAtex disappears in the urine, soon after the treatment of the disease and this is one of the advantages of this test. KAtex is an easy-performing method which takes only a few minutes to perform the test. For KAtex, cross-reacting antigens are present in the urine of VL patients and also healthy individuals. Urine samples must be boiled (in boiling water) to eliminate the cross-reacting antigens. Attempt has been made to eliminate this step, by treating the samples with different chemicals, but the outcome has not been so satisfactory [12].

The diagnostic accuracy of KAtex has been evaluated in different VL-endemic areas with a 
relatively diverse range of specificity and sensitivity [9,12-19]. In an appropriately comprehensive study, KAtex along with freeze-dried direct agglutination test (FD-DAT) and the rK39 dipstick were evaluated in the Indian subcontinent and East Africa for the diagnosis of VL [9]. Results showed a sensitivity and specificity of 84 and 87.8 in Kenya, 71 and 64\% in Ethiopia, 66.1 and 87.6 in India, 35.8 and 97.8 in Nepal, and 72.9 and 98.3 in Sudan [9]. However, the study has not included all the relevant literatures about the KAtex and a few exciting studies about KAtex are available afterward.

KAtex showed a reasonable diagnostic performance for the diagnosis of VL in HIV/VL coinfected cases [18]. A monoclonal antibody raised against the KAtex target antigen showed a sensitivity of $94.1 \%$ and specificity of $100 \%$ when the monoclonal antibody was used in a capture ELISA for the diagnosis of VL [20].

While KAtex has been considered as a promising test for the diagnosis of VL and has been evaluated in different VL-endemic areas, a global assessment of this test is needed. The current meta-analysis was performed to evaluate the diagnostic accuracy of KAtex for the diagnosis of VL.

\section{Methodology}

All articles published after the introduction of latex agglutination test for the diagnosis of VL (2000), and when KAtex become available until March 2017 were searched.

\section{Selection criteria}

The inclusion criteria were visceral leishmaniasis (not CL, MCL or PKDL), human subjects, and availability of the absolute numbers of true positive, true negative, false positive and false negative in the presented data. Studies which evaluated the HIV/VL co-infection were also included. Those articles which reported other diagnostic tools (i. e., latex agglutination test for detection of anti-leishmanial antibodies) were excluded. Unavailability of control group or proper reference test was also considered as the exclusion criteria. Besides, studies which evaluated fewer than 10 VL patients' samples were excluded.

\section{Literature review}

All the related published literature cited within PubMed, ISI web of science, Google Scholar, Scopus, and Iranmedex were searched. The search terms, both as $\mathrm{MeSH}$ terms and text words, were "visceral leishmaniasis", "Leishmaniasis", 'Kala-azar' combined with "KAtex", "latex agglutination test", or "urinary antigen". The reference lists of the retrieved studies were also sought for additional studies. This initial search generated 57 articles. No duplicates were found. Following reading the title and/or abstract, of 57 articles, 30 studies were excluded and following reading the full text, 10 more articles were excluded because they did not meet the inclusion criteria. Thus, 17 studies met the eligibility criteria which constituted the basis of this meta-analysis.

\section{Assessment of study quality}

Quada-2 (quality assessment of diagnostic accuracy studies) tools were used for quality assessment of the included studies. Quality assessment was done by BS and MF and discrepancies, was resolved by consensus after discussion.

\section{Data extraction}

From each study, the following items were extracted from the full-length articles. (I) number of VL patients, (II) number of controls, (III) number of nonVL patients (patients with other diseases), (IV) sensitivity and specificity of the test, (V), positive predictive value (PPV) and negative predictive value (NPV), if presented, (VI) country where the sample originated (VII) reference test, (VIII) number of true positive, true negative, false positive and false negative. PPV and NPV were calculated in cases which were not present, based on the presented data. Moreover, the confidence interval for sensitivity and specificity were estimated from the available data when the studies did not provide them. Data were extracted from selected papers into structured tables containing all the descriptive and relevant variables.

\section{Statistical analysis}

R 3.2.2 was used to do the analysis. Meta-analysis of diagnostic accuracy (MADA) and Hierarchical Summary Receiver Operating Curve (HSROC) packages were used in order to do the meta-analysis and to obtain pooled estimates of sensitivity and specificity. Sensitivity, specificity, PPV, NPV, and OR are expressed by forest plots. Summary receiver operating characteristics (SROC) plot is also provided. Fixed effect bivariate analysis was conducted, using MantelHaenszel estimator, to measure the performance and diagnosis odds ratio (DOR), of the test. Heterogeneity of the test results was assessed by Chi-squared test and is presented by forest plot. 


\section{Results}

Flowchart of the included studies is presented in Figure 1. Seventeen studies evaluating the latex agglutination test (KAtex) met the inclusion criteria and data from these studies were included in the metaanalysis. In some studies, (e.g. Attar et al., 2001), samples had been collected from different geographical areas with related controls. Therefore, diagnostic performances were calculated based on the presented data [10]. The 17 articles, included 23 studies (i.e., more than one group of VL patients evaluated per article). The studies included a total of $2623 \mathrm{VL}$ patients and 2350 controls. The main reference test in most of the studies was a positive parasitological detection of Leishmania amastigotes in spleen, lymph nodes or bone marrow samples. Table 1 summarizes the characteristics of the included studies.

Sensitivity ranged from 39.8 to $100 \%$ and specificity ranged from 64 to $100 \%$. PPV and NPV ranged from 73.6 to $100 \%$ and 39.8 to $100 \%$, respectively.
Figure 1. Flowchart of the included studies.

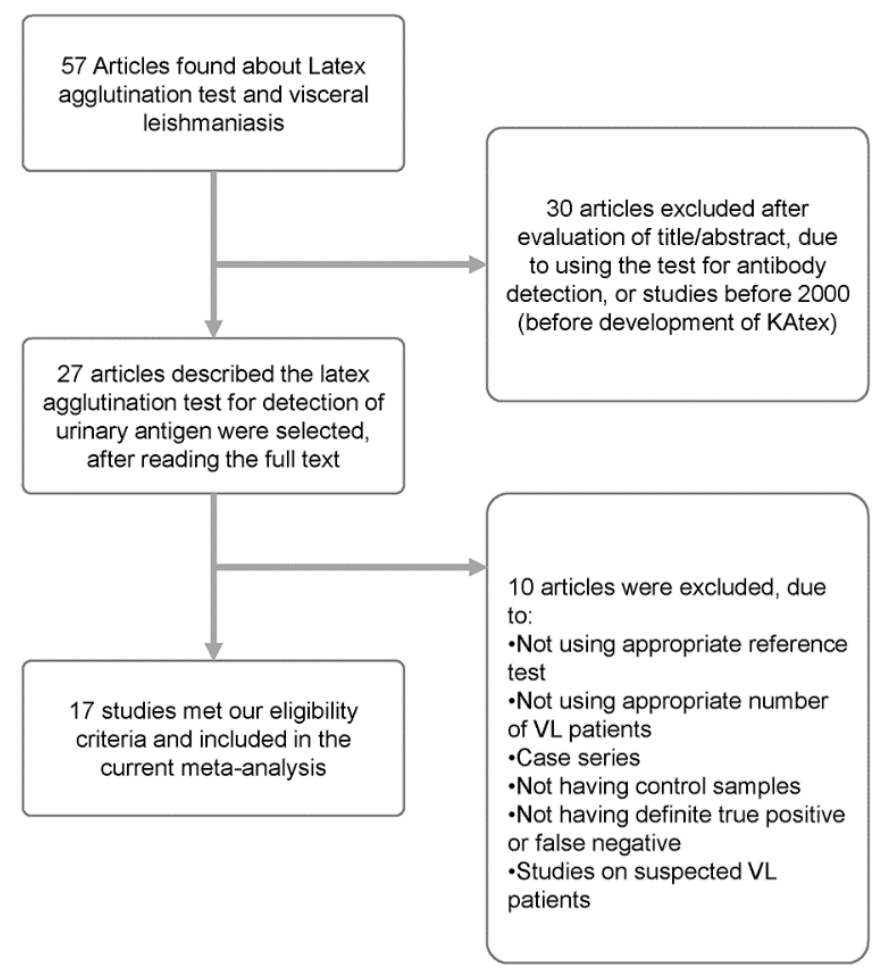

Table 1. Characteristics of the included studies.

\begin{tabular}{|c|c|c|c|c|c|c|c|c|}
\hline \multicolumn{2}{|c|}{ No. of subjects } & \multirow{2}{*}{$\begin{array}{l}\text { Sensitivity } \\
\text { (\%) (CI) * }\end{array}$} & \multirow{2}{*}{$\begin{array}{l}\text { Specificity } \\
\text { (\%) (CI) }\end{array}$} & \multirow{2}{*}{$\begin{array}{c}\text { PPV } \\
(\%)(C I)\end{array}$} & \multirow{2}{*}{$\begin{array}{c}\text { NPV } \\
(\%)(C I)\end{array}$} & \multirow[b]{2}{*}{ Year } & \multirow[b]{2}{*}{ Author (Ref No.) } & \multirow[b]{2}{*}{ Country } \\
\hline $\begin{array}{c}\text { VL } \\
\text { patients }\end{array}$ & Controls & & & & & & & \\
\hline 230 & 170 & 73.5 (67.1-78.9) & 99 (93.7-99.9) & 99.4 (96.2-99.9) & $61.8(53.8-69.3)$ & 2007 & Sundar [25] & India \\
\hline 382 & 185 & $87(83.3-90.3)$ & 99 (95.7-100) & $99.4(97.6-100)$ & $78.8(72.9-83.8)$ & 2005 & Sundar [26] & India \\
\hline 29 & 90 & 82.7 (67.4-95.4) & 98.9 (93-99.9) & 86.8 (78.4-99.7) & 99.7 (88.7-98.6) & 2002 & Molai [28] & Iran \\
\hline 36 & 40 & 75 (57-87) & $100(89-100)$ & $100(84.4-100)$ & $81.6(67.4-90.7)$ & $2007-2008$ & Salam [29] & Bangladesh \\
\hline 155 & 77 & $47.7(39.7-55.9)$ & $98.7(93-100)$ & $98.7(92.8-100)$ & $48.4(40.4-56.4)$ & 2001-2002 & Rijal [21] & Nepal \\
\hline $\begin{array}{l}\text { HIV co } \\
\text { infection } \\
(\mathrm{n}: 49)\end{array}$ & 59 & $85.7(72-93.5)$ & $100(92.3-100)$ & $100(89.5-100)$ & $89.4(78.7-95.2)$ & $1994-2003$ & Riera [30] & Spain \\
\hline 31 & 61 & 83.9 (65.5-93.9) & $100(92.6-100)$ & $100(83.9-100)$ & $92.4(82.4-97.1)$ & $2005-2007$ & Ghatei [16] & Iran \\
\hline 18 & 71 & 77.7 (51.9-92.6) & 98.3 (91.4-99.9) & $93.3(66-99.6)$ & $93.3(86.2-98.3)$ & 2008 & Gavgani [15] & Iran \\
\hline 62 & 194 & $95.2(85.6-98.7)$ & $100(97-100)$ & $100(92.3-100)$ & $98.1(94.2-99.5)$ & 2001-2002 & El-safi [31] & $\begin{array}{l}\text { Eastern } \\
\text { Sudan }\end{array}$ \\
\hline 85 & 57 & $57(46-67)$ & $98(88-100)$ & $98(89-100)$ & $56(45-67)$ & 2005 & Chappuis [27] & Nepal \\
\hline 294 & 183 & $72.9(63.4-81.7)$ & $98.3(95.3-99.8)$ & 96.9 (88.8-99) & $86.1(80.5-90.3)$ & 2003 & Boelaert [9] & Sudan \\
\hline 38 & 15 & $71.0(52.0-87.1)$ & $64.0(40.0-84.6)$ & $73.6(48.5-89.8)$ & $62.5(38.5-83.7)$ & 2004 & Boelaert [9] & Ethiopia \\
\hline 308 & 120 & $84.5(78.6-89.8)$ & $87.8(80.8-93.5)$ & $91.3(85.8-94.8)$ & $78.3(70.2-84.8)$ & 2004-2005 & Boelaert [9] & Kenya \\
\hline 352 & 72 & $66.1(60.4-71.6)$ & $87.6(79.1-94.1)$ & $95.3(91.1-97.7)$ & $39.8(32.2-47.9)$ & 2005 & Boelaert [9] & India \\
\hline 158 & 72 & $35.8(27.5-44.7)$ & $97.8(92.1-100)$ & $95.3(91.1-97.7)$ & $39.8(32.2-47.9)$ & 2005 & Boelaert [9] & Nepal \\
\hline 25 & 369 & $64(42.6-81.2)$ & $100(98.7-100)$ & $100(75.9-100)$ & $79(63.5-89.4)$ & 2001 & Attar [10] & Brazil \\
\hline 29 & 369 & $86.2(67.4-95.4)$ & $100(98.7-100)$ & $100(83.4-100)$ & $98.8(96.7-99.6)$ & 2001 & Attar [10] & Yemen \\
\hline 15 & 47 & $100(98.7-100)$ & $88.6(76.2-95.3)$ & $71.4(47.6-87.8)$ & $100(89.3-100)$ & 2001 & Attar [10] & Sudan \\
\hline 12 & 73 & $100(98.7-100)$ & $96(88-98.9)$ & $84.2(59.5-95.8)$ & $100(93.7-100)$ & 2004 & Vilaplana [32] & _ \\
\hline 100 & 50 & $87(78.4-92.6)$ & $100(91.1-100)$ & & $79.3(66-88.1)$ & $2007-8$ & Habib [17] & Bangladesh \\
\hline 50 & 50 & $94(82.4-98.4)$ & $98(87.9-99.8)$ & $97.91(87.5-99.8)$ & $94.23(83.1-98.5$ & 2008 & Ahsan [13] & Bangladesh \\
\hline 35 & 62 & $51.4(34.3-68.3)$ & $98.3(90.2-99.9)$ & 94.7 (71.8-99.7) & $78.2(67.1-86.4)$ & 2016 & Ben-Abid [22] & Tunisia \\
\hline 150 & 305 & $87(80.6-92)$ & $98(97-99.8)$ & 98.4 (94.1-99.7) & $94(90.7-96.3) 35$ & 2010 & Singh [33] & India \\
\hline
\end{tabular}

* VL: Visceral Leishmaniasis; PPV: Positive Predictive Value; NPV: Negative Predictive Value; CI: 95\% Confidence Interval. 
Figure 2. Individual and pooled sensitivity estimates of KAtex for the diagnosis of VL.

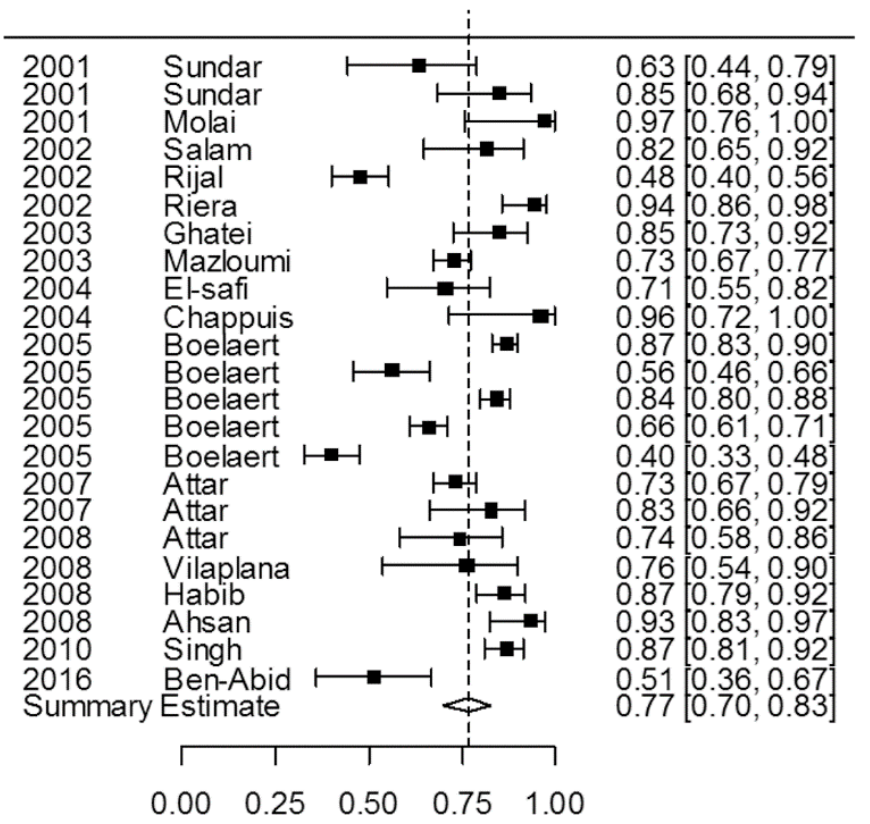

Figure 4. Individual and pooled PPV estimates of KAtex for the diagnosis of VL.

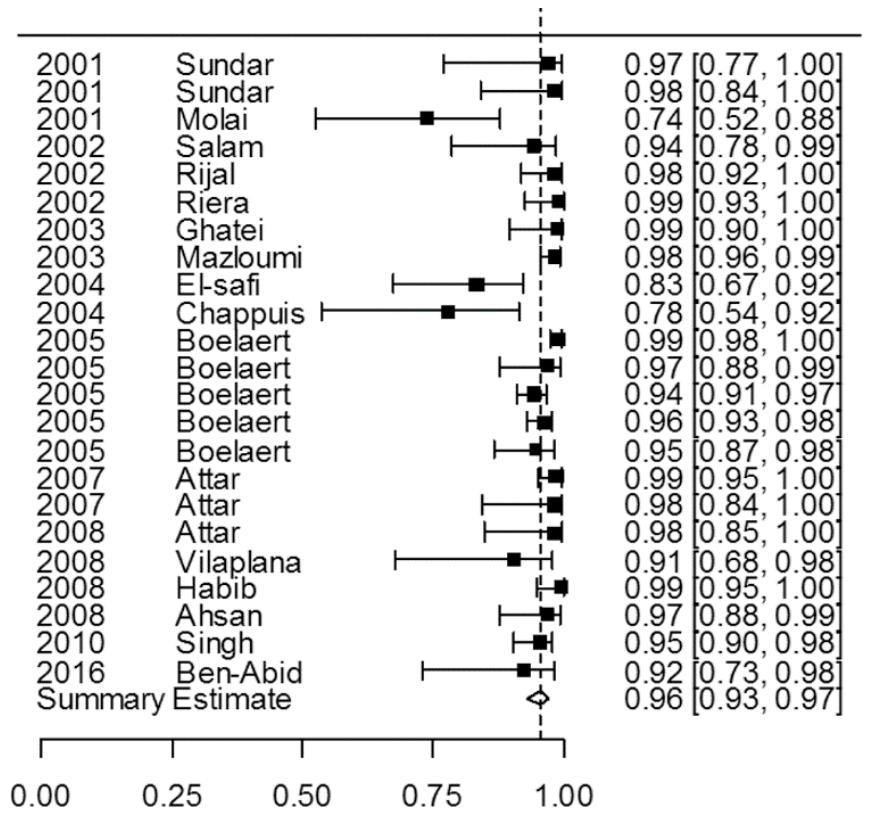

Figure 3. Individual and pooled specificity estimates of KAtex for the diagnosis of VL.

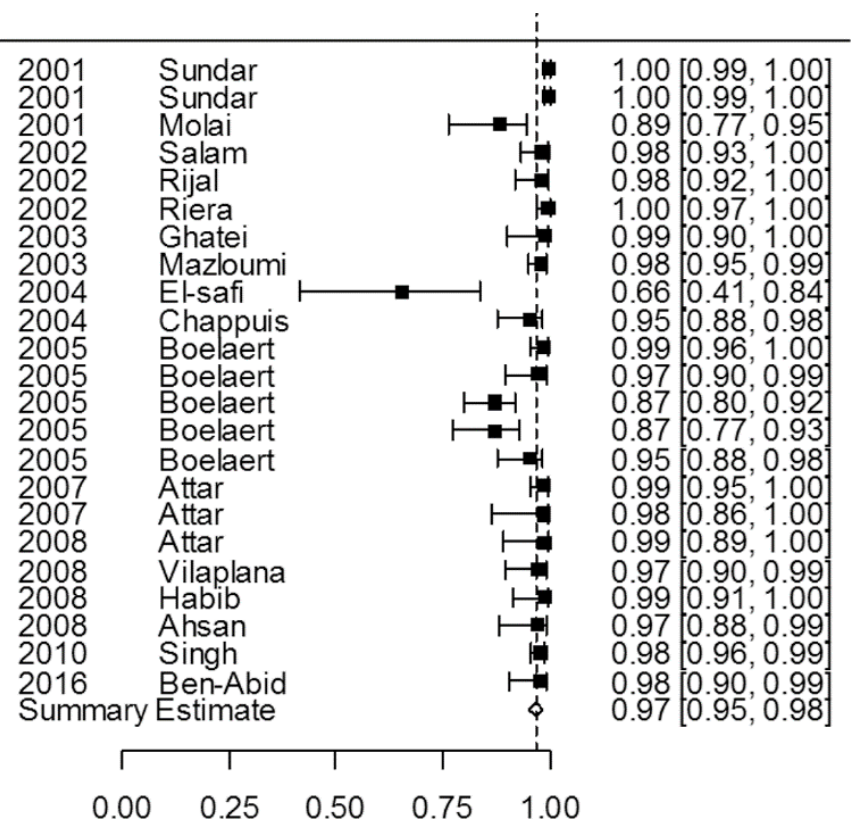

Figure 5. Individual and pooled NPV estimates of KAtex for the diagnosis of VL.

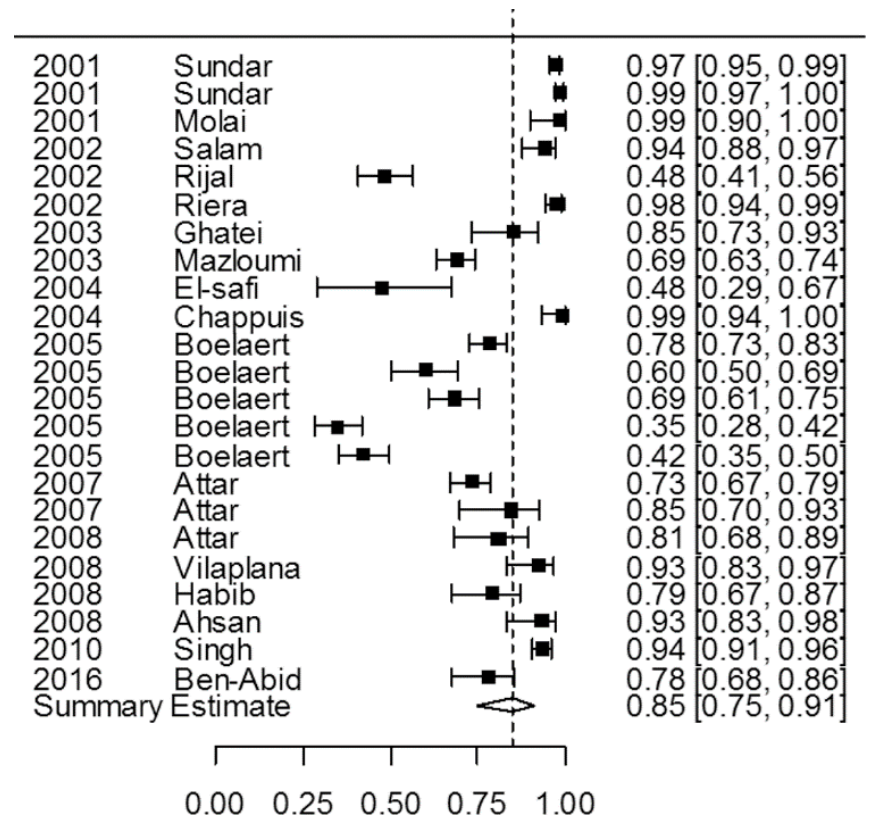


The pooled estimated sensitivity and specificity of KAtex were 77\% (95: CI: 70-83\%) and 97\% (95\% CI: 95-98\%) respectively. Moreover the summary estimate of PPV and NPV for KAtex were 96\% (95\% CI: 9397\%) and 85\% (95\% CI: 75-91\%) respectively. Figure 2 and 3 show the results of individual and pooled sensitivity and specificity estimates and Figure 4 and 5 shows the PPV and NPV for KAtex. Diagnostic odds ratio (DOR) of KAtex is depicted in Figure 6 and SROC curve for KAtex is illustrated in Figure 7. Comparing the performance of the test by region suggests a significant difference. Accordingly, the lowest and highest sensitivities are reported from Nepal/Tunisia and Europe/Middle East respectively $(p<0.05)$. On the other hand, the lowest and highest rates of specificity were reported from Sudan and America/Middle East respectively. With regard to positive and negative predictive values, again studies from the Middle East reported the highest rates $(p<0.05)$. Figure 8 shows the individual and summary estimates of sensitivity, specificity, NPV, and PPV of KAtex, based on region.

\section{Discussion}

There are quite numbers of papers which evaluated the diagnostic performance of KAtex for the diagnosis of VL. Findings of the current meta-analysis revealed that KAtex has a reasonable and appropriate specificity $(97 \%)$ in the diagnosis of VL. However, the summary estimate of the sensitivity of KAtex in this metaanalysis was $77 \%$, which is somewhat far from appropriateness. On the other hand, KAtex has got a reasonable sensitivity in some of the VL-endemic areas

Figure 7. The SROC curve for KAtex in the diagnosis of VL $(\mathrm{AUC}=0.94)$.

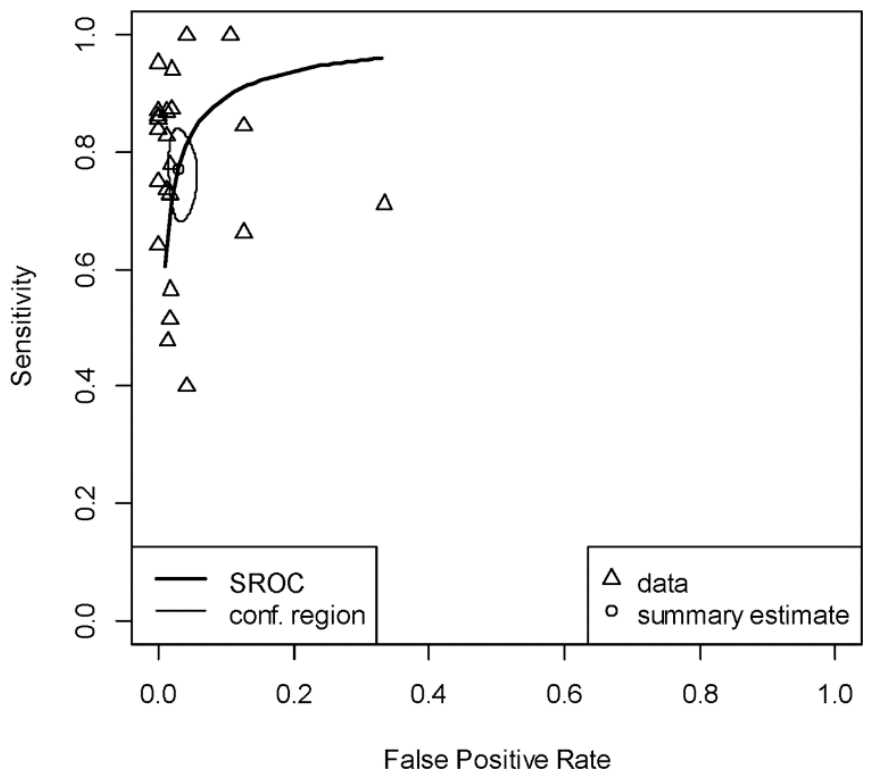

Figure 6. The diagnostic Odd ratio of KAtex.

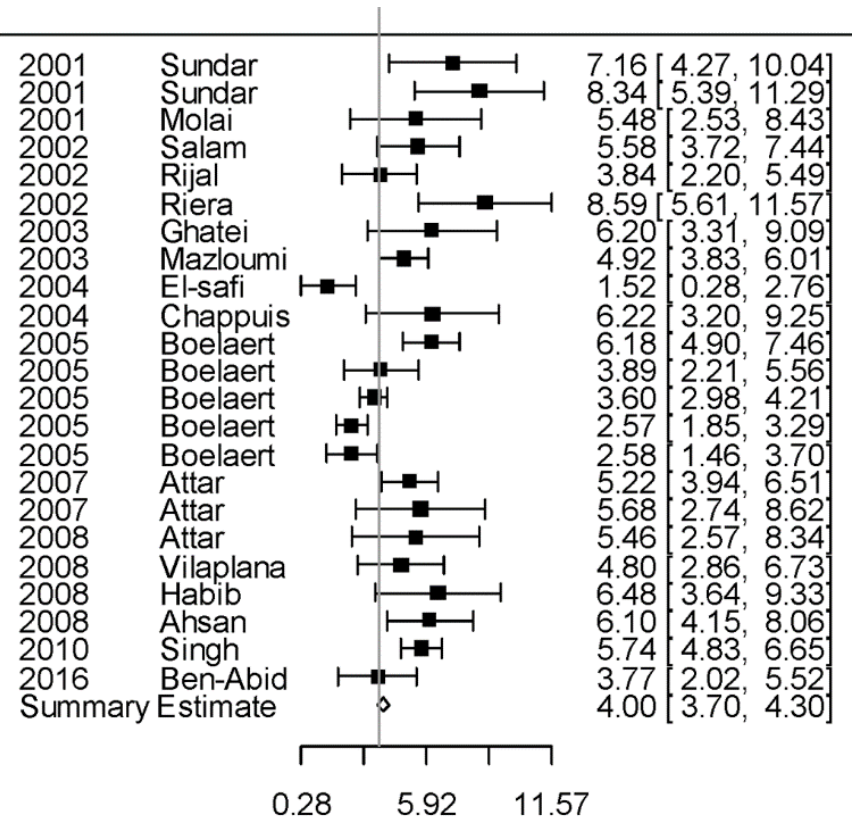

log diagnostic odds ratio

of the world, including the Middle East and Europe. Very low sensitivity has been recorded for KAtex in Nepal and Tunisia [21-22]. Discrepancies in the sensitivity and specificity of the test may be linked to the participated patients (severity of the disease and parasite and also antigen load), geographical region of the study, and lack of homogeneity of the studied population.

KAtex is the most studied antigen detection assay for the diagnosis of VL. KAtex's target antigen has

Figure 8. Individual and summary estimates of sensitivity, specificity, NPV, and PPV of KAtex, based on region.

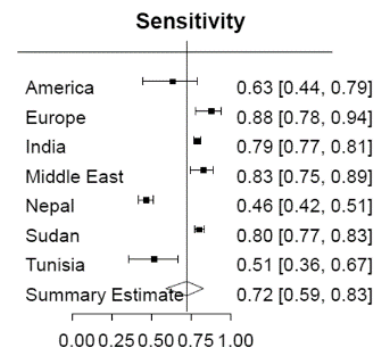

Positive predictive value

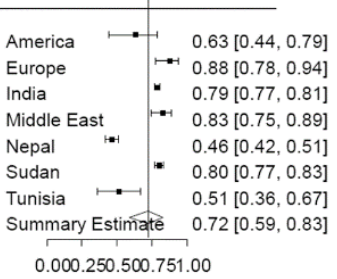

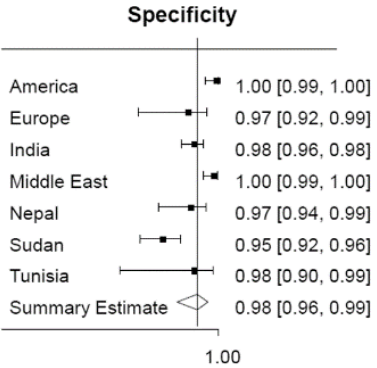

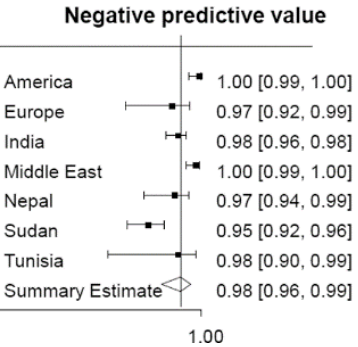


found to be a carbohydrate compound which seems to be linked to the LPG of Leishmania [11]. KAtex has a very good prognostic value as the urinary antigen disappears within a few weeks of successful treatment.

Recently a few polypeptides antigens have been detected in the urine of VL patients with promising diagnostic performance [23]. Three distinct $L$. infantum parasite antigens including $L$. infantum iron superoxidase 1 and $L$. infantum nuclear transport factor 2 have been reported by Abeijon et al. [23]. Antibodies which have been produced against these antigens were applied in a standard ELISA system and a sensitivity of $89 \%$ and specificity of $100 \%$ were reported for the assay [24]. Utilizing the combination of the three protein antigens in an ELISA system showed 100 sensitivity and specificity when urine samples of $20 \mathrm{VL}$ patients were tested by the assay [24]. However, the assay evaluated only small samples of VL patients and much more trials in the field are necessary to find out the actual accuracy of this assay for the diagnosis of VL. Furthermore, the assay is based on the detection of only $L$. infantum antigen and may not accurately detect the antigen of $L$. donovani, which is the main causative agent of VL in the Old World [24]. This is actually not the case for KAtex, as KAtex can detect antigen in the urine of VL patients caused by either L. infantum ( $L$. chagasi) or L. donovani. Therefore, it can be said that KAtex is not species specific. Simultaneous detection of a panel of urinary antigen, both polypeptide and carbohydrate antigen, might be a reasonable approach for improving the sensitivity of urinary antigen-based detection assays. This requires the coating of latex particles with a cocktail of antibodies raised against currently recognized polypeptides and carbohydrate antigens.

In this metanalysis, due to the low number of studies in some regions, including Spain and Tunisia, only one study per region was included in the analysis. By no means, this is one of the limitations of the current study. The imperfection of the reference standard test is another limitation of this study which may be present in other similar studies about the performances of the diagnostic tests in the diagnosis of VL [19].

Taken together, findings of the current metaanalysis demonstrated that KAtex has a relatively high specificity for the diagnosis of VL in different VLendemic areas, but its sensitivity is far from satisfactory. As KAtex is a simple, field applicable, easy performing and cost-effective test, improvement of its sensitivity deserve further studies.

\section{Acknowledgements}

The study was financially supported by the office of vicechancellor for research of Shiraz University of Medical Sciences (Grant No. 14052).

\section{Author Contributions}

BS performed the literature search and assessed the included studies, wrote the first draft of the manuscript, edited and approved the final version. SAK, ZR, and SNG performed the literature and approved the final version of the manuscript. MF helped with the assessment of included studies, performed the data statistical analysis and approved the final version of the manuscript.

\section{References}

1. Postigo JA (2010) Leishmaniasis in the World Health Organization Eastern Mediterranean Region. Int J Antimicrob Agents 36 Suppl 1: 62-65.

2. Salam N, Al-Shaqha WM, Azzi A (2014) Leishmaniasis in the Middle East: incidence and epidemiology. PLoS Negl Trop Dis 8: e3208.

3. Sarkari B, Hatam G, Ghatee MA (2012) Epidemiological features of visceral leishmaniasis in Fars province, southern Iran. Iran J Public Health 41: 94-99.

4. Sarkari B, Naraki T, Ghatee MA, Abdolahi Khabisi S, Davami MH (2016) Visceral leishmaniasis in Southwestern Iran: A retrospective clinico-hematological analysis of 380 consecutive hospitalized cases (1999-2014). PLoS One 11: e0150406.

5. Hatam G, Mikaeili F, Sadjjadi S, Sarkari B (2007) Direct agglutination test and enzyme-linked immunosorbent assay with urine samples for the diagnosis of visceral leishmaniasis. Iran J Parasitol 2: 24-28.

6. Mikaeili F, Fakhar M, Sarkari B, Motazedian MH, Hatam G (2007) Comparison of serological methods (ELISA, DAT, and IFA) for diagnosis of visceral leishmaniasis utilizing an endemic strain. Iran J Immunol 4: 116-121.

7. Sarkari B, Ashrafmansouri M, Hatam G, Habibi P, Abdolahi Khabisi S (2014) Performance of an ELISA and indirect immunofluorescence assay in serological diagnosis of zoonotic cutaneous leishmaniasis in Iran. Interdiscip Perspect Infect Dis 2014: 505134.

8. Sarkari B, Hatam G, Mikaeili F, Sadeghi H, Ebrahimi S (2008) A comparative study of antigen and antibody detection in visceral leishmaniasis using serum and urine-based ELISA. Trop Biomed 25: 96-99.

9. Boelaert M, El-Safi S, Hailu A, Mukhtar M, Rijal S, Sundar S, Wasunna M, Aseffa A, Mbui J, Menten J, Desjeux P, Peeling RW (2008) Diagnostic tests for kala-azar: a multi-center study of the freeze-dried DAT, rK39 strip test and KAtex in East Africa and the Indian subcontinent. Trans R Soc Trop Med Hyg 102: $32-40$.

10. Attar ZJ, Chance ML, el-Safi S, Carney J, Azazy A, El-Hadi M, Dourado C, Hommel M (2001) Latex agglutination test for the detection of urinary antigens in visceral leishmaniasis. Acta Trop 78: 11-16.

11. Sarkari B, Chance M, Hommel M (2002) Antigenuria in visceral leishmaniasis: detection and partial characterisation of a carbohydrate antigen. Acta Trop 82: 339-348.

12. Hatam GR, Ghatee MA, Hossini SM, Sarkari B (2009) Improvement of the newly developed latex agglutination test 
(KAtex) for diagnosis of visceral leishmaniasis. J Clin Lab Anal 23: 202-205.

13. Ahsan MM, Islam MN, Mollah AH, Hoque MA, Hossain MA, Begum Z, Islam MT (2010) Evaluation of latex agglutination test (KAtex) for early diagnosis of kala-azar. Mymensingh Med J 19: 335-339.

14. Diro E, Techane Y, Tefera T, Assefa Y, Kebede T, Genetu A, Kebede Y, Tesfaye A, Ergicho B, Gebre-Yohannes A, Mengistu G, Engers H, Aseffa A, Desjeux P, Boelaert M, Hailu A (2007) Field evaluation of FD-DAT, rK39 dipstick and KATEX (urine latex agglutination) for diagnosis of visceral leishmaniasis in northwest Ethiopia. Trans R Soc Trop Med Hyg 101: 908-914.

15. Gavgani AM, Vatan SK, Ghazanchaei A (2008) KAtex antigen-detection test as a diagnostic tool for latent visceral leishmaniasis cases. African J Biotechnol 7: 852-859.

16. Ghatei MA, Hatam GR, Hossini MH, Sarkari B (2009) Performance of latex agglutination test (KAtex) in diagnosis of visceral leishmaniasis in Iran. Iran J Immunol 6: 202-207.

17. Habib ZH, Lutfor AB, Jhora ST, Ahmed I, Akhter H (2015) Validity of KAtex test for the diagnosis of visceral leishmaniasis in endemic region of Bangladesh. Bangladesh J Infect Dis 1: 8-11.

18. Riera C, Fisa R, Lopez P, Ribera E, Carrió J, Falcó V, Molina I, Gállego M, Portús M. (2004) Evaluation of a latex agglutination test (KAtex) for detection of Leishmania antigen in urine of patients with HIV-Leishmania coinfection: value in diagnosis and post-treatment follow-up. Eur J Clin Microbiol Infect Dis 23: 899-904.

19. Boelaert M, Verdonck K, Menten J, Sunyoto T, van Griensven J, Chappuis F, Rijal S (2014) Rapid tests for the diagnosis of visceral leishmaniasis in patients with suspected disease. Cochrane Database Syst Rev: Cd009135.

20. Sarkari B, Chance M, Hommel M (2005) A capture ELISA for the diagnosis of visceral leishmaniasis using a monoclonal antibody against a leishmanial urinary antigen. Iranian Biomed J 9: 117-122.

21. Rijal S, Boelaert M, Regmi S, Karki BM, Jacquet D, Singh R, Chance ML, Chappuis F, Hommel M, Desjeux P, Van der Stuyft P, Le Ray D, Koirala S (2004) Evaluation of a urinary antigen-based latex agglutination test in the diagnosis of kalaazar in eastern Nepal. Trop Med Int Health 9: 724-729.

22. Ben-Abid M, Galaï Y, Habboul Z, Ben-Abdelaziz R, BenSghaier I, Aoun K, Bouratbine A (2017) Diagnosis of Mediterranean visceral leishmaniasis by detection of Leishmania-related antigen in urine and oral fluid samples. Acta Trop 167: 71-72.

23. Abeijon C, Kashino SS, Silva FO, Costa DL, Fujiwara RT, Costa CH, Campos-Neto A (2012) Identification and diagnostic utility of Leishmania infantum proteins found in urine samples from patients with visceral leishmaniasis. Clin Vaccine Immunol 19: 935-943.

24. Abeijon C, Campos-Neto A (2013) Potential non-invasive urine-based antigen (protein) detection assay to diagnose active visceral leishmaniasis. PLoS Negl Trop Dis 7: e2161.
25. Sundar S, Singh RK, Bimal SK, Gidwani K, Mishra A Maurya R, Singh SK, Manandhar KD, Boelaert M, Rai M (2007) Comparative evaluation of parasitology and serological tests in the diagnosis of visceral leishmaniasis in India: a phase III diagnostic accuracy study. Trop Med Int Health 12: 284-289.

26. Sundar S, Agrawal S, Pai K, Chance M, Hommel M (2005) Detection of leishmanial antigen in the urine of patients with visceral leishmaniasis by a latex agglutination test. Am J Trop Med Hyg 73: 269-271.

27. Chappuis F, Rijal S, Jha UK, Desjeux P, Karki BM, Koirala S, Loutan L, Boelaert M (2006) Field validity, reproducibility and feasibility of diagnostic tests for visceral leishmaniasis in rural Nepal. Trop Med Int Health 11: 31-40.

28. Molai S, Mohebali M, Akhoundi B, Zarei Z (2006) Evaluation of latex agglutination test (KATEX $®)$ for the detection of urinary antigens in human visceral leishmaniasis. J School Public Health Institute Public Health Res 4: 1-8.

29. Salam M, Khan MG, Mondal D (2011) Urine antigen detection by latex agglutination test for diagnosis and assessment of initial cure of visceral leishmaniasis. Trans R Soc Trop Med Hyg 105: 269-272.

30. Riera C, Fisa R, Lopez P, Ribera E, Carrió J, Falcó V, Molina I, Gállego M, Portús M (2004) Evaluation of a latex agglutination test (KAtex) for detection of Leishmania antigen in urine of patients with HIV-Leishmania coinfection: value in diagnosis and post-treatment follow-up. Eur J Clin Microbiol Infect Dis 23: 899-904.

31. El-Safi SH, Abdel-Haleem A, Hammad A, El-Basha I, Omer A, Kareem HG, Boelaert M, Chance M, Hommel M (2003) Field evaluation of latex agglutination test for detecting urinary antigens in visceral leishmaniasis in Sudan. East Mediterr Health J 9: 844-855.

32. Vilaplana C, Blanco S, Domínguez J, Giménez M, Ausina V, TUral C, Muñoz C (2004) Noninvasive method for diagnosis of visceral leishmaniasis by a latex agglutination test for detection of antigens in urine samples. J Clin Microbiol 42: 1853-1854.

33. Singh DP, Goyal RK, Singh RK, Sundar S, Mohapatra TM (2010) In search of an ideal test for diagnosis and prognosis of kala-azar. J Health Popul Nutr 28: 281-285.

\section{Corresponding author}

Bahador Sarkari, PhD

Department of Parasitology and Mycology,

School of Medicine,

Shiraz University of Medical Sciences,

Shiraz, Iran

P. O. Box: 71345-1735

Tel: 98-71-32305291

Fax: 98-71-32305291

Email: sarkarib@sums.ac.ir

Conflict of interests: No conflict of interests is declared. 